\title{
Sequence Characterization of 3-Dimensional Riordan Arrays and Some Application
}

\author{
Roksana Słowik(i)
}

\begin{abstract}
We propose the characterization of 3-dimensional Riordan arrays with use of three sequences that is analogous to the representation of 2-dimensional Riordan arrays with use of $A$ and $Z$-sequence. We also suggest an application of this representation for finding totally positive matrices.
\end{abstract}

Mathematics Subject Classification. Primary 05A15, 15B99, 15B05, 05 A05.

Keywords. Riordan group, multi-dimensional Riordan array, 3-dimensional Riordan array, Riordan array, sequence characterization, $A$-sequence, $Z$-sequence.

\section{Introduction}

Let's recall that the Riordan group, introduced in [1], is a group of $\mathbb{N}_{0} \times$ $\mathbb{N}_{0}$ matrices that are identified with pairs of formal power series. Namely, denoting by $\mathcal{F}_{0}$ - the ring of formal power series with nonzero free term, and by $\mathcal{F}_{1}$ - the ring of formal power series with zero free term but nonzero the next term, the Riordan group $\mathcal{R}$ consists of pairs $\mathcal{R}(g, f)$ with $g \in \mathcal{F}_{0}, f \in \mathcal{F}_{1}$. The multiplication of these pairs is given by

$$
\mathcal{R}\left(g_{1}(z), f_{1}(z)\right) * \mathcal{R}\left(g_{2}(z), f_{2}(z)\right)=\mathcal{R}\left(g_{1}(z) \cdot g_{2}\left(f_{1}(z)\right), f_{2}\left(f_{1}(z)\right)\right),
$$

and it coincides with multiplication of corresponding matrices.

Recently, one can observe an interest in multi-dimensional matrix algebra [2-4]. Here we would like to focus on three-dimensional matrices. The $(2,1)$ product $C=\left[c_{i j k}\right]$ of $A=\left[a_{i j k}\right], B=\left[b_{i j k}\right]$, is defined by the formula:

$$
c_{i j k}=\sum_{x \geq 0} a_{i x k} b_{x j k} .
$$


In this note we are interested in $\mathcal{R}^{\langle 3\rangle}$ — the group of 3-dimensional Riordan arrays. It was proved by Cheon and Jin [5] that $\mathcal{R}^{\langle 3\rangle}$ is an extension of $\mathcal{R}$ by $\mathcal{F}_{0}$. In this group the matrices $R=\left[r_{n k m}\right]_{n, k, m \in \mathbb{N}_{0}}$ are associated with the triples of series $(g, f, h)$ with $g, h \in \mathcal{F}_{0}, f \in \mathcal{F}_{1}$. The multiplication of such triples is defined as follows:

$$
\begin{gathered}
\mathcal{R}\left(g_{1}(z), f_{1}(z), h_{1}(z)\right) * \mathcal{R}\left(g_{2}(z), f_{2}(z), h_{2}(z)\right) \\
= \\
\mathcal{R}\left(g_{1}(z) \cdot g_{2}\left(f_{1}(z)\right), f_{2}\left(f_{1}(z)\right), h_{1}(z) \cdot h_{2}\left(f_{1}(z)\right)\right),
\end{gathered}
$$

Each entry of $R=\left[r_{n k m}\right]$ can be found from the relation:

$$
r_{n k m}=\left[z^{n}\right] g f^{k} h^{m},
$$

where $\left[z^{n}\right] f$ denotes the $n$-th coefficient in the series expansion of $f$.

Thanks to definition (1.3), multiplication (1.2) corresponds with matrix multiplication given by (1.1).

It is known (see $[6,7]$ ) that Riordan arrays can be uniquely determined by two sequences, called $A$-sequence and $Z$-sequence. More precisely, starting with $r_{00}=g_{0}$, all the other entries can be found using the relations:

$$
\begin{aligned}
r_{n+1, k+1} & =a_{0} r_{n k}+a_{1} r_{n, k+1}+a_{2} r_{n, k+2}+\cdots, \\
r_{n+1,0} & =z_{0} r_{n 0}+z_{1} r_{n 1}+z_{2} r_{n 2}+\cdots .
\end{aligned}
$$

In this paper we wish to give an analogous presentation for 3-dimensional Riordan arrays. Namely, we propose $A, Z$, and $H$-sequence, that completely characterize Riordan array:

$$
\begin{aligned}
r_{n 00} & =z_{0} r_{n-1,0,0}+z_{1} r_{n-1,1,0}+z_{2} r_{n-1,2,0}+\cdots \\
r_{n k 0} & =a_{0} r_{n-1, k+1,0}+a_{1} r_{n-1, k+2,0}+a_{2} r_{n-1, k+3,0}+\cdots \\
r_{n k m} & =h_{0} r_{n, k, m-1}+h_{1} r_{n-1, k, m-1}+h_{2} r_{n-2, k, m-1}+\cdots .
\end{aligned}
$$

We will show that the following theorem is true.

Theorem 1.1. Any 3-dimensional Riordan array is completely characterized by its $A, Z$ and $H$ sequence given as in (1.5). Moreover

$$
f(z)=z \cdot A(f(z)), \quad g(z)=\frac{g_{0}}{1-z \cdot Z(f(z))}, \quad H(z)=h(z) .
$$

After discussing the above presentation, we will propose how it can be used to obtain some totally positive Riordan arrays.

\section{The Disscussion}

\subsection{Sequence Characterization}

To prove the main result, it suffices to notice that the below lemma holds.

Lemma 2.1. The groups

$$
\text { 1. } \mathcal{R}_{g}^{\langle 3\rangle}:=\left\{(g, f, 1): g \in \mathcal{F}_{0}, f \in \mathcal{F}_{1}\right\},
$$


2. $\mathcal{R}_{h}^{\langle 3\rangle}:=\left\{(1, f, h): h \in \mathcal{F}_{0}, f \in \mathcal{F}_{1}\right\}$

are isomorphic with $\mathcal{R}$. Moreover

1. $A$ and $Z$-sequence of $\mathcal{R}(g, f, 1)$ coincide with $A$ and $Z$-sequence of $\mathcal{R}(g, f)$,

2. $H$ and $Z$-squence of $\mathcal{R}(1, f, h)$ coincide with $A$ and $Z$-sequence of $\mathcal{R}(h, f)$. Proof. Clearly, the maps $\phi_{g}: \mathcal{R}_{g}^{\langle 3\rangle} \rightarrow \mathcal{R}, \phi_{h}: \mathcal{R}_{h}^{\langle 3\rangle} \rightarrow \mathcal{R}$ given by

$$
\phi_{g}(\mathcal{R}(g, h, 1))=(g, h), \quad \phi_{h}(\mathcal{R}(1, f, h))=(h, f)
$$

establish the desired isomorphism, and the correspondence of sequences.

Proof of Theorem 1.1. Comparing (1.4) and (1.5) one can notice that $A$ and $Z$-sequence of $\mathcal{R}(g, f, h)$ coincide with $A$ and $Z$-sequence of $\mathcal{R}(g, f)$. Thus, we only need to check the last equality. Using (1.3) we get

$$
\begin{aligned}
r_{n k m} & =\left[z^{n}\right] g f^{k} h^{m}=\sum_{i=0}^{n}\left(\left[z^{i}\right] g f^{k} \cdot\left[z^{n-i}\right] h\right)=\sum_{i=0} r_{i, k, m-1} \cdot h_{n-i} \\
& =\sum_{j=0}^{n} h_{j} r_{n-j, k, m-1} .
\end{aligned}
$$

For 2-dimensional Riordan arrays the following result was obtained by He and Sprugnoli.

Theorem 2.2 [8, Thm.3.3,3.4]. Let $\mathcal{R}\left(g_{1}, f_{1}\right), \mathcal{R}\left(g_{2}, f_{2}\right)$ be 2-dimensional Riordan arrays with $A, Z$-sequences: $A_{1}, Z_{1}$ and $A_{2}, Z_{2}$, respectively. Then $A$ and $Z$-sequence of the product $\mathcal{R}\left(g_{1}, f_{1}\right) * \mathcal{R}\left(g_{2}, f_{2}\right)$ is equal to

$$
\begin{gathered}
A(z)=A_{2}(z) \cdot A_{1}\left(\frac{z}{A_{2}(z)}\right) \\
Z(z)=\left(1-\frac{z}{A_{2}(z)} Z_{2}(z)\right) \cdot Z_{1}(z)+A_{1}\left(\frac{z}{A_{2}(z)}\right) \cdot Z_{2}(z) .
\end{gathered}
$$

Based on the above one can prove the following.

Proposition 2.3. Let $\mathcal{R}\left(g_{1}, f_{1}, h_{1}\right), \mathcal{R}\left(g_{2}, f_{2}, h_{2}\right)$ be 3-dimensional Riordan arrays with $A, Z$ and $H$-sequences: $A_{1}, Z_{1}, H_{1}$ and $A_{2}, Z_{2}, H_{2}$, respectively. Then $A, Z$ and $H$-sequence of the product $\mathcal{R}\left(g_{1}, f_{1}, h_{1}\right) * \mathcal{R}\left(g_{2}, f_{2}, h_{2}\right)$ is equal to

$$
\begin{aligned}
& A(z)=A_{2}(z) \cdot A_{1}\left(\frac{z}{A_{2}(z)}\right) \\
& Z(z)=\left[1-\frac{z}{A_{2}(z)} Z_{2}(z)\right] \cdot Z_{1}\left(\frac{z}{A_{2}(z)}\right)+A_{1}\left(\frac{z}{A_{2}(z)}\right) \cdot Z_{2}(z) \\
& H(z)=H_{2}(z) \cdot H_{1}\left(\frac{z}{H_{2}(z)}\right) .
\end{aligned}
$$


Proof. By Lemma 2.1, $A$ and $Z$-sequence of $\mathcal{R}(g, f)$ coincide with $A$ and $Z$ sequence of $\mathcal{R}(g, f, h)$, so two first equalities hold. By Theorem $1.1, h$-sequence of $\mathcal{R}(g, f, h)$ is equal to $h$, so from the definition and Lemma 2.1, we get that $h$-sequence of $\mathcal{R}\left(g_{1}(z) \cdot g_{2}\left(f_{1}(z)\right), f_{2}\left(f_{1}(z)\right), h_{1}(z) \cdot h_{2}\left(f_{1}(z)\right)\right)$ is the same as $A$-sequence of

$$
\mathcal{R}\left(h_{1}(z) \cdot h_{2}\left(f_{1}(z)\right), f_{2}\left(f_{1}(z)\right)\right)=\mathcal{R}\left(h_{1}(z), f_{1}(z)\right) * \mathcal{R}\left(h_{2}(z), f_{2}(z)\right) .
$$

Thus, using Theorem 2.2 again, we get the third equality.

\subsection{Possible Application}

In this section we join the representation proposed in the first section with some other issue. Namely, the total positivity of a Riordan matrix. An infinite matrix is said to be totally positive (or shortly $T P$ ) if all its minors are nonnegative. In particular, a Toeplitz matrix

$$
\left[\begin{array}{ccccc}
a_{0} & & & & \\
a_{1} & a_{0} & & & \\
a_{2} & a_{1} & a_{0} & & \\
a_{3} & a_{2} & a_{1} & a_{0} & \\
\vdots & & & & \ddots
\end{array}\right] \quad \text { with all } a_{n} \geq 0
$$

is totally positive if and only if $a(z)=\sum_{n=0}^{\infty} a_{n} z^{n}$ has only real (and nonpositive) zeros, and in this case $\left(a_{n}\right)_{n=0}^{\infty}$ is called Pólya frequency sequence.

Let's get back to our matrices. It is obvious that fixing $m$ in (1.3), one obtains a 2-dimensional Riordan array. It is called the $m$-th layer of $\mathcal{R}(g, f, h)$. According to (1.3), the $m$-th layer of $\mathcal{R}(g, f, h)$ is equal to $\mathcal{R}\left(g h^{m}, f\right)$. From

$\mathcal{R}\left(g(z) h^{m}(z), f(z)\right)=\mathcal{R}\left(h^{m}(z), z\right) * \mathcal{R}(g(z), f(z))=(\mathcal{R}(h(z), z))^{m} * \mathcal{R}(g(z), f(z))$,

and the fact that the product of $T P$ matrices is a $T P$ matrix, we get the following conclusion.

Corollary 2.4. If $\mathcal{R}(g(z), f(z))$ and $\mathcal{R}(h(z), z)$ are totally positive, then every layer of $\mathcal{R}(g(z), f(z), h(z))$ is totally positive.

Totally positive matrices were considered in the context of $A$ and $Z$ sequences.

It was first proved in [9] (see also $[10,11]$ ) that every $2 D$ Riordan array $\mathcal{R}(g, f)$ is induced by its production matrix

$$
P_{\mathcal{R}(g, f)}=\left[\begin{array}{cccccc}
z_{0} & a_{0} & & & \\
z_{1} & a_{1} & a_{0} & & \\
z_{2} & a_{2} & a_{1} & a_{0} & \\
\vdots & & & & \ddots
\end{array}\right] .
$$


In particular, if we write $U$ (as in [11]) for the shift matrix:

$$
U=\left[\begin{array}{ccccc}
0 & 1 & & & \\
0 & 0 & 1 & & \\
0 & 0 & 0 & 1 & \\
\vdots & & & & \ddots
\end{array}\right],
$$

then $P_{\mathcal{R}(g, f)}$ is the production matrix of the Riordan array $\mathcal{R}(g, f)$ if and only if $U \mathcal{R}(g, f)=\mathcal{R}(g, f) P_{\mathcal{R}(g, f)}$.

From [12] we know that if the production matrix $P_{\mathcal{R}(g, f)}$ is $T P$, then $\mathcal{R}(g, f)$ is $T P$ as well. Thus, we finish with the following observation.

Corollary 2.5. If $P_{\mathcal{R}(g, f)}$ given by (2.1) is TP matrix and $H$ is a Pólya frequency sequence, then every layer of $\mathcal{R}(g, f, h)$ is a totally positive matrix.

Example. It can be checked that for

$$
A=(2,3,1,0,0,0,0, \ldots), \quad Z=(3,5,0,0,0,0, \ldots)
$$

the production matrix $P_{\mathcal{R}(g, f)}$ is $T P$ (see [12]). Moreover,

$$
H=(2,5,4,1,0,0,0,0, \ldots)
$$

is a Pólya frequency sequence. Thus, all the layers of $\mathcal{R}(g, f, h)$

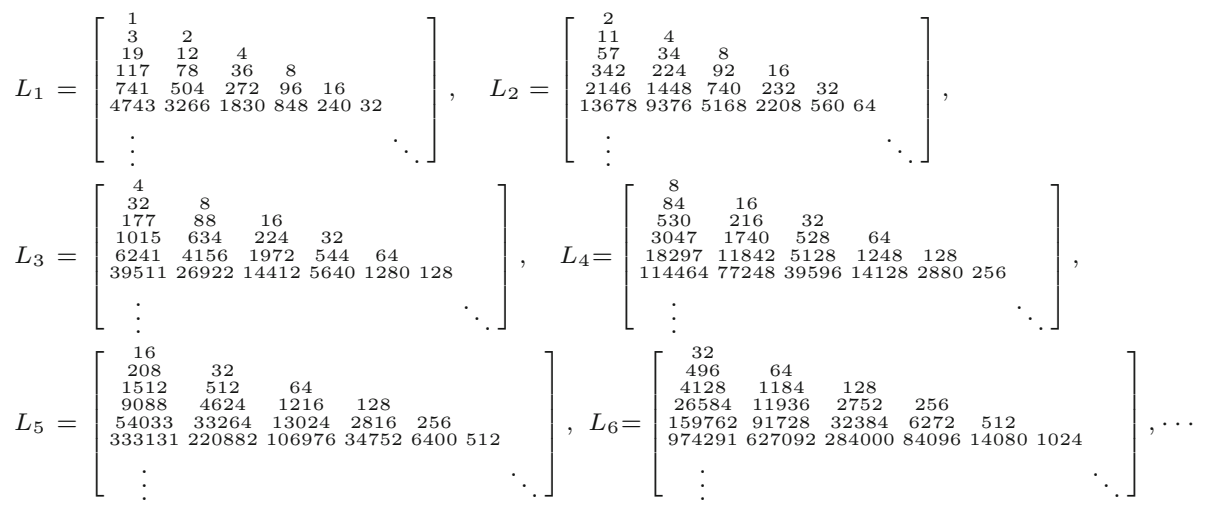

are totally positive.

\section{Some Closing Comments}

Let's finish this short note with some remarks about possible generalizations of the presented notions. In [13] the authors proposed extending the definition of 2-dimensional Riordan array given by

$$
r_{n k}=\left[z^{n}\right] g f^{k} \quad n, k \in \mathbf{N}_{0}
$$

to all $n, k \in \mathbb{Z}$, and called them recursive matrices (in [14] they are also called complementary). Also in 3-dimensional case one can introduce 3-dimensional recursive matrix ${ }_{\mathbb{Z}} R=\left[r_{n k m}\right]$ whose entries are given by (1.3) for all $n, k, m \in$ 
$\mathbb{Z}$. It is interesting that, by [15] (see Section 3 of this paper) for such $\mathbb{Z} R$ the following identities hold:

$$
\begin{aligned}
r_{n+m, k+m, p} & =\sum_{j=0}^{n-k} a_{j}^{(m)} r_{n, k+j, p}, \\
r_{n+m, k+m, p} & =\sum_{j=0}^{n-k} f_{j+m}^{(m)} r_{n-j, k, p},
\end{aligned}
$$

where by $a_{j}^{(m)}$ we mean $\left[z^{j}\right] A^{m}$.

\section{Acknowledgements}

The author would like to thank anonymous referee for his/her remarks.

Open Access. This article is distributed under the terms of the Creative Commons Attribution 4.0 International License (http://creativecommons.org/licenses/ by/4.0/), which permits unrestricted use, distribution, and reproduction in any medium, provided you give appropriate credit to the original author(s) and the source, provide a link to the Creative Commons license, and indicate if changes were made.

\section{References}

[1] Shapiro, L.W., Getu, S., Woan, W.-J., Woodson, L.: The Riordan group. Discrete Appl. Math. 34, 229-239 (1991)

[2] Solo, A.M.G.: Multidimensional matrix mathematics: notation, representation, and simplification, Part 1 of 6 . In: Proceedings of the World Congress on Engineering 2010, vol. III (2010)

[3] Solo, A.M.G.: Multidimensional matrix mathematics: multidimensional matrix equality, addition, substraction and multiplication, Part 2 of 6. In: Proceedings of the World Congress on Engineering 2010, vol. III (2010)

[4] Solo, A.M.G.: Multidimensional matrix mathematics: algebraic laws, Part 5 of 6. In: Proceedings of the World Congress on Engineering 2010, vol. III (2010)

[5] Cheon, G.-S., Jin, S.-T.: The group of multidimensional Riordan arrays. Linear Algebra Appl. 524, 263-277 (2017)

[6] Rogers, D.G.: Pascal triangles, Catalan numbers and renewal arrays. Discrete Math. 22, 301-310 (1978)

[7] Merlini, D., Rogers, D.G., Sprugnoli, R., Verri, M.C.: On some alternative characterizations of Riordan arrays. Can. J. Math. 49, 301-320 (1997)

[8] He, T.-X., Sprugnoli, R.: Sequence characterization of Riordan arrays. Discrete Math. 309, 3962-3974 (2009)

[9] Merlini, D., Verri, M.C.: Generating trees and proper Riordan arrays. Discrete Math. 218(1-3), 167-183 (2000) 
[10] Deutsch, E., Ferrari, L., Rinaldi, S.: Production matrices and Riordan arrays. Ann. Comb. 13, 65-85 (2009)

[11] He, T.-X.: Matrix characterizations of Riordan arrays. Linear Algebra Appl. 465, 15-42 (2015)

[12] Chen, X., Liang, H., Wang, Y.: Total positivity of Riordan arrays. Eur. J. Comb. 46, 68-74 (2015)

[13] Barnabei, M., Brini, A., Nioletti, G.: Recursive matrices and umbral calculus. J. Algebra 75, 546-573 (1982)

[14] Luzón, A., Merlini, D., Morón, M.A., Sprugnoli, R.: Complementary Riordan arrays. Discrete Appl. Math. 172, 75-87 (2014)

[15] Luzón, A., Merlini, D., Morón, M.A., Sprugnoli, R.: Identities induced by Riordan arrays. Linear Algebra Appl. 436, 631-647 (2012)

Roksana Słowik

Institute of Mathematics

Silesian University of Technology

Kaszubska 23

44-100 Gliwice

Poland

e-mail: roksana.slowik@gmail.com

Received: March 4, 2019.

Accepted: August 20, 2019.

Publisher's Note Springer Nature remains neutral with regard to jurisdictional claims in published maps and institutional affiliations. 